Article

\title{
The Lanczos Equation on Light-Like Hypersurfaces in a Cosmologically Viable Class of Kinetic Gravity Braiding Theories
}

\author{
Bence Racskó ${ }^{1 \text { (ID }}$ and László Á. Gergely ${ }^{2, *}$ (D) \\ 1 Department of Theoretical Physics, University of Szeged, Tisza L. krt. 84-86, H-6720 Szeged, Hungary; \\ daeron806@gmail.com \\ 2 Institute of Physics, University of Szeged, Dóm tér 9, H-6720 Szeged, Hungary \\ * Correspondence: laszlo.a.gergely@gmail.com or gergely@physx.u-szeged.hu
}

Received: 11 April 2019; Accepted: 22 April 2019; Published: 2 May 2019

\begin{abstract}
We discuss junction conditions across null hypersurfaces in a class of scalar-tensor gravity theories (i) with second-order dynamics, (ii) obeying the recent constraints imposed by gravitational wave propagation, and (iii) allowing for a cosmologically viable evolution. These requirements select kinetic gravity braiding models with linear kinetic term dependence and scalar field-dependent coupling to curvature. We explore a pseudo-orthonormal tetrad and its allowed gauge fixing with one null vector standing as the normal and the other being transversal to the hypersurface. We derive a generalization of the Lanczos equation in a $2+1$ decomposed form, relating the energy density, current, and isotropic pressure of a distributional source to the jumps in the transverse curvature and transverse derivative of the scalar. Additionally, we discuss a scalar junction condition and its implications for the distributional source.
\end{abstract}

Keywords: scalar-tensor gravity; junction conditions; null hypersurfaces

\section{Introduction}

Scalar-tensor gravity theories give viable modifications of general relativity in which accelerated expansion could be recovered without dark energy at late times; well-tested solar system constraints could be obeyed (for example through the Vainshtein mechanism); and the recent constraint from gravitational wave detection [1-7] on the propagation speed of the tensorial modes could be successfully implemented. Indeed, from the class of Hordeski theories ensuring second-order dynamics for both the scalar field and the metric tensor [8,9], a subclass has been identified [10-13] in which gravitational waves propagate with the speed of light (as verified both from the almost coincident detection with accompanying $\gamma$-rays in the case of the neutron star binary merger and from a stringent test of the dispersion relations disruling massive modes for the 10 black hole mergers). This subclass contains cubic derivative couplings of the scalar field in the Lagrangian, known as kinetic gravity braiding $[14,15]$. In the Jordan frame, the curvature couples with the scalar through an unspecified function of the scalar field.

This class of scalar-tensor gravity models could be further restricted by the requirement to ensure a viable cosmological evolution. In Ref. [16], it has been proven that for a kinetic gravity braiding model with Lagrangian only linearly and quadratically depending on the kinetic term $X=-(\nabla \phi)^{2} / 2$, an autonomous system of equations governs the dynamics, leading to a number of fixed points for the background dynamics, with three of them representing consecutive radiation-, matter-, and dark energy-dominated regimes (see for example Figure 1 of Ref. [16]). The same model was further analyzed from the string theory-motivated point of view of avoiding de Sitter regimes, which are 
not embeddable in string theory [17]. Cross-correlating this model class with the requirement of the propagation of tensorial modes with the speed of light, the quadratic dependence has to be dropped. In this paper, we consider this class of kinetic gravity braiding models with only linear dependence on the kinetic terms and analyze the junctions across null hypersurfaces.

Junction conditions in general relativity are known either for spatial or temporal hypersurfaces [18] or for null hypersurfaces $[19,20]$. The latter are more sophisticated, as the normal to the hypersurface is not suitable for a $3+1$ space-time decomposition, being also tangent at the same time. The decomposition can be done with respect to a transverse vector, with the gauge arising from its non-unique choice dropping out from the final results [19], or by employing a pseudo-orthonormal basis with two null vectors, one of them playing the role of the normal, the other being transversal [20]. The distributional contribution arising in the curvature from the possible discontinuity of the metric derivative across the hypersurface is related to singular sources on the hypersurface through the Lanczos equation. The same technique led to the derivation of the dynamics on a brane embedded in a 5-dimensional bulk [21-23].

In the full Horndeski class of scalar-tensor gravity theories, junction conditions across spatial or temporal hypersurfaces have been derived [24,25], but the null case stays uncovered, despite its importance being undoubted as all electromagnetic and gravitational shock-waves propagate along such hypersurfaces.

Here we propose to derive such junction conditions for the class of kinetic gravity braiding theories with a linear kinetic terms, which, as discussed above, are both cosmologically viable and obey the gravitational wave constraints. This generalizes our earlier work on null junctions in Brans-Dicke theories [26].

The notations are as follows: space-time indices are Greek, 2-dimensional spatial indices are Latin capital letters. The soldering of any quantity $A$, with values $A^{+}$and $A^{-}$on the two sides of the hypersurface, is $\tilde{A}=A^{+} \Theta(f)+A^{-} \Theta(-f)$, where $\Theta$ is the step function. The average on the hypersurface is denoted as $\langle A\rangle=\left(A^{+}+A^{-}\right) / 2$ and the jump over the hypersurface as $[A]=$ $A^{+}-A^{-}$.

\section{Equations of Motion}

The assumed Lagrangian

$$
L_{G K G B}=\underbrace{B(\phi) X+V(\phi)}_{L_{2}} \underbrace{-2 \xi(\phi) \square \phi X}_{L_{3}} \underbrace{\frac{1}{2} F(\phi) R}_{L_{4}}
$$

with $B, \xi, F$ arbitrary functions of the scalar field yields the following expressions through the variation of metric

$$
\begin{aligned}
& E_{\mu \nu}^{(2)}=-\frac{1}{2} B(\phi)\left(X g_{\mu \nu}-\phi_{\mu} \phi_{v}\right)-\frac{1}{2} V(\phi) g_{\mu v}, \\
& E_{\mu \nu}^{(3)}=\xi(\phi) \square \phi \phi_{\mu} \phi_{v}+2 \xi^{\prime}(\phi) X\left(\phi_{\mu} \phi_{v}+X g_{\mu \nu}\right)+2 \xi(\phi) X_{(\mu} \phi_{v)}-\xi(\phi) X_{\kappa} \phi^{\kappa} g_{\mu v}, \\
& E_{\mu \nu}^{(4)}=\frac{1}{2}\left\{F(\phi) G_{\mu v}+\left(F^{\prime}(\phi) \square \phi-2 F^{\prime \prime}(\phi) X\right) g_{\mu v}-F^{\prime}(\phi) \phi_{\mu v}-F^{\prime \prime}(\phi) \phi_{\mu} \phi_{\nu}\right\},
\end{aligned}
$$

and through the variation of the scalar field

$$
\begin{aligned}
E_{\phi}^{(2)} & =B(\phi) \square \phi-B^{\prime}(\phi) X+V^{\prime}(\phi), \\
E_{\phi}^{(3)} & =\xi(\phi)\left\{(\square \phi)^{2}-\phi_{\mu v} \phi^{\mu v}-R^{\mu v} \phi_{\mu} \phi_{v}\right\}-2 \xi^{\prime \prime}(\phi) X^{2}, \\
E_{\phi}^{(4)} & =\frac{1}{2} F^{\prime}(\phi) R,
\end{aligned}
$$


where $\phi_{\mu} \equiv \nabla_{\mu} \phi$ and $\phi_{\mu v} \equiv \nabla_{\nu} \nabla_{\mu} \phi$. The Ricci curvature tensor appears in the expression $E_{\phi}^{(3)}$ through the Ricci identity $\left[\nabla_{\mu}, \nabla_{\nu}\right] V^{\kappa}=R^{\kappa}{ }_{\lambda \mu v} V^{\lambda}$, which has been used to get rid of third derivatives of $\phi$.

These are the left-hand sides of the equations of motion (EoMs). The right-hand sides are half of the energy-momentum tensor for the metric variation of the matter action and zero for the scalar field variation, as in the Jordan frame the matter does not couple to the scalar field.

\section{Junction Conditions}

\subsection{The Extrinsic Formulation}

We employ a pseudo-orthonormal basis with two null vectors $N^{\mu}$ and $L^{\mu}$, the first being the normal (surface gradient, which is also tangent) to the hypersurface $\Sigma$ and the other playing the role of the transverse vector, with respect to which we perform a $(2+1)+1$ decomposition [20]. The normalization is $L^{\mu} N_{\mu}=-1$. The continuity of both the metric tensor $g_{\mu \nu}$ and scalar $\phi$ are imposed over the hypersurface: $[\phi]=\left[g_{\mu v}\right]=0$. Their first derivatives in the null transverse direction $\phi_{L} \equiv L^{\mu} \partial_{\mu} \phi$ and $L^{\rho} \partial_{\rho} g_{\mu \nu}$ may have a jump

$$
\zeta=\left[\phi_{L}\right], \quad c_{\mu v}=\left[L^{\rho} \partial_{\rho} g_{\mu v}\right],
$$

and since all tangential derivatives are assumed to be continuous, we have

$$
\left[\phi_{\mu}\right]=-N_{\mu} \zeta, \quad\left[\partial_{\kappa} g_{\mu v}\right]=-N_{\kappa} c_{\mu v} .
$$

The second-order derivatives appearing in the equations of motion

$$
\begin{aligned}
E_{\mu v} & \equiv \tilde{E}_{\mu v}+\mathscr{E}_{\mu \nu} \delta(f)=\frac{1}{2}\left(\tilde{T}_{\mu v}+\mathscr{T}_{\mu \nu} \delta(f)\right), \\
E_{\phi} & \equiv \tilde{E_{\phi}}+\mathscr{E}_{\phi} \delta(f)=0
\end{aligned}
$$

lead to the distributional contributions $\mathscr{E}_{\mu v}$ and $\mathscr{E}_{\phi}$ along the thin shell, arising from the derivative of the step function. All quantities with a tilde are the regular contributions to the respective quantities. For consistency, we also include a distributional energy-momentum tensor $\mathscr{T}_{\mu v}$ together with the regular one $\tilde{T}_{\mu v}$. In the argument of the delta distribution, $f$ denotes a function which generates the hypersurface as its zero set. For convenience, we also assume that $N_{\mu}=\nabla_{\mu} f$.

We introduce the notations

$$
c_{\mu}=c_{\mu v} N^{v}, \quad c^{\dagger}=c_{\mu} N^{\mu}, \quad c=c_{\mu}^{\mu}
$$

and explicitly give the jump of the connection as

$$
\left[\Gamma_{\mu v}^{\kappa}\right]=-\frac{1}{2}\left(N_{\mu} c_{v}^{\kappa}+N_{\nu} c_{\mu}^{\kappa}-N^{\kappa} c_{\mu \nu}\right)
$$

hence the singular parts of the curvature tensor and its traces become

$$
\begin{aligned}
\mathscr{R}^{\kappa}{ }_{\lambda \mu \nu} & =-\frac{1}{2}\left(N_{\mu} c_{\nu}^{\kappa} N_{\lambda}-N_{\nu} c_{\mu}^{\kappa} N_{\lambda}+N_{\nu} c_{\mu \lambda} N^{\kappa}-N_{\mu} c_{\nu \lambda} N^{\kappa}\right), \\
\mathscr{R}_{\mu \nu} & =-\frac{1}{2}\left(N_{\mu} c_{\nu}+N_{\nu} c_{\mu}-N_{\mu} N_{\nu} c\right), \\
\mathscr{R} & =-c^{\dagger} .
\end{aligned}
$$

In particular, the singular part of the Einstein tensor is

$$
\mathscr{G}_{\mu v}=-\frac{1}{2}\left(N_{\mu} c_{\nu}+N_{\nu} c_{\mu}-N_{\mu} N_{\nu} c-c^{\dagger} g_{\mu v}\right) .
$$


We also give the jumps and singular parts of the quantities constructed from the scalar field. As a calligraphic version of $\phi$ is not catchy, in the decomposition $A=\tilde{A}+\mathscr{A} \delta(f)$ we introduce the alternative notation $\mathscr{A} \equiv \operatorname{Sing}(A)$, denoting the singular part of the arbitrary quantity $A$.

For the scalar field, we have

$$
\operatorname{Sing}\left(\phi_{\mu v}\right)=-\zeta N_{\mu} N_{v}, \quad \operatorname{Sing}(\square \phi)=-\zeta N_{\mu} N^{\mu}=0, \quad[X]=\phi_{N} \zeta,
$$

where $\phi_{N}=N^{\mu} \phi_{\mu}$ is the normal derivative. We note that the value of $\phi_{N}$ on the hypersurface is unambigous, being a tangential derivative, which is continuous.

Explicit calculation gives the hypersurface contributions to the left-hand side of the tensorial EoMs:

$$
\begin{aligned}
& \mathscr{E}_{\mu \nu}^{(2)}=0, \\
& \mathscr{E}_{\mu \nu}^{(3)}=\xi(\phi) \zeta\left(2 \phi_{N} N_{(\mu}\left\langle\phi_{v)}\right\rangle-\phi_{N}^{2} g_{\mu \nu}\right), \\
& \mathscr{E}_{\mu \nu}^{(4)}=\frac{1}{2}\left(F(\phi) \mathscr{G}_{\mu v}+F^{\prime}(\phi) \zeta N_{\mu} N_{\nu}\right)
\end{aligned}
$$

and the hypersurface contributions to the left-hand side of the scalar EoMs:

$$
\begin{aligned}
& \mathscr{E}_{\phi}^{(2)}=0, \\
& \mathscr{E}_{\phi}^{(3)}=\xi(\phi)\left(2 \zeta N^{\mu} N^{v}\left\langle\phi_{\mu \nu}\right\rangle+\phi_{N} c^{\mu}\left\langle\phi_{\mu}\right\rangle-\frac{1}{2} \phi_{N}^{2} c\right), \\
& \mathscr{E}_{\phi}^{(4)}=-\frac{1}{2} F^{\prime}(\phi) c^{\dagger} .
\end{aligned}
$$

\subsection{The Intrinsic Formulation}

The above equations are expressed in a four-dimensional coordinate system smooth across the hypersurface. Such coordinate systems may be difficult to construct, hence it would be more practical to use coordinantes intrinsic to the junction hypersurface.

The hypersurface contributions to the left-hand side of the tensor EoMs (20) and (21) are tangential in the sense that

$$
\mathscr{E}_{\mu v}^{(3)} N^{v}=\mathscr{E}_{\mu v}^{(4)} N^{v}=0
$$

Hence, we may expand them in a basis adapted to the junction hypersurface $\Sigma$. We choose this basis as $\left(L^{\mu}, N^{\mu}, e_{2}^{\mu}, e_{3}^{\mu}\right)$, where the $e_{A}^{\mu}$ are two spacelike tangent vector fields to $\Sigma$, satisfying

$$
N_{\mu} e_{A}^{\mu}=L_{\mu} e_{A}^{\mu}=0 .
$$

For a fixed choice of $N^{\mu}$, we may always choose $e_{A}^{\mu}$ such that the vector fields $\left(N^{\mu}, e_{2}^{\mu}, e_{3}^{\mu}\right)$ form a holonomic set, but this is not imperative (we may also choose them to form a pseudo-orthonormal system). The following statements are also valid in the anholonomic case. The inner products of the spacelike vectors generate a spacelike induced metric

$$
q_{A B}=g_{\mu \nu} e_{A}^{\mu} e_{B}^{v}
$$

on the two-dimensional subspaces spanned by the vectors $e_{A}^{\mu}$. Its inverse is denoted $q^{A B}$ (capital Latin indices are raised and lowered by either the metric or its inverse). The completeness relation of the adapted basis is

$$
g^{\mu v}=-L^{\mu} N^{v}-N^{\mu} L^{v}+q^{A B} e_{A}^{\mu} e_{B}^{v} .
$$


We further denote $e_{1}^{\mu}=N^{\mu}$, with the Latin indices $a, b, \ldots$ taking the values $1,2, \ldots$. The extrinsic curvature $K_{a b}=e_{a}^{\mu} e_{b}^{v} \frac{1}{2} \mathcal{L}_{N} g_{\mu \nu}$ is unsuitable to describe the transversal change in the metric as $N^{\mu}$ is also tangential. For this reason, we introduce the transverse curvature [20]:

$$
\mathcal{K}_{a b}=\frac{1}{2} e_{a}^{\mu} e_{b}^{v} \mathcal{L}_{L} g_{\mu v}
$$

with its jump related to $c_{\mu v}$ as

$$
\left[\mathcal{K}_{a b}\right]=\frac{1}{2} e_{a}^{\mu} e_{b}^{v} c_{\mu \nu}
$$

The singular part (hypersurface contribution) of the Einstein equation is but the generalized Lanczos equation

$$
\mathscr{E}^{\mu \nu}=\frac{1}{2} \mathscr{T}^{\mu \nu},
$$

where $\mathscr{E}$ is the sum of the terms (20) and (21). As the left-hand side is purely tangential, the distributional stress-energy-momentum tensor admits the decomposition

$$
\mathscr{T}^{\mu v}=\rho N^{\mu} N^{v}+j^{A}\left(N^{\mu} e_{A}^{v}+e_{A}^{\mu} N^{v}\right)+p^{A B} e_{A}^{\mu} e_{B}^{v}
$$

where $\rho, j^{A}$, and $p^{A B}$ are the energy density, current vector, and stress tensor of the distributional source, respectively. These quantities, defined as the components emerging with respect to the intrinsic triad of vectors, can be evaluated even when the bulk coordinates do not match smoothly along $\Sigma$. They are defined as

$$
\rho=2 \mathscr{E}_{\mu v} L^{\mu} L^{v}, \quad j_{A}=-2 \mathscr{E}_{\mu v} L^{\mu} e_{A}^{v}, \quad p^{A B}=2 \mathscr{E}_{\mu \nu} e_{A}^{\mu} e_{B}^{v}
$$

The $2+1$ decomposition of Equation (31) yields an isotropic pressure $p^{A B}=p q^{A B}$ and

$$
\begin{aligned}
\rho & =F(\phi)\left[\mathcal{K}_{A B}\right] q^{A B}+F^{\prime}(\phi)\left[\phi_{L}\right]-2 \xi(\phi) \phi_{N}\left[\phi_{L}^{2}\right], \\
j_{A} & =-F(\phi)\left[\mathcal{K}_{N A}\right]+2 \xi(\phi)\left[\phi_{L}\right] \phi_{N} \phi_{A}, \\
p & =F(\phi)\left[\mathcal{K}_{N N}\right]-2 \xi(\phi)\left[\phi_{L}\right] \phi_{N^{\prime}}^{2}
\end{aligned}
$$

where $\phi_{A}=e_{A}^{\mu} \phi_{\mu}, \mathcal{K}_{N A} \equiv \mathcal{K}_{1 A}$, and $\mathcal{K}_{N N} \equiv \mathcal{K}_{11}$.

The scalar equation is

$$
\begin{aligned}
0= & \xi(\phi) \phi_{N}^{2} q^{A B}\left[\mathcal{K}_{A B}\right]-2 \xi(\phi) \phi_{N} \phi^{A}\left[\mathcal{K}_{N A}\right] \\
& +\left(F^{\prime}(\phi)+2 \xi(\phi) \phi_{N}\left\langle\phi_{L}\right\rangle\right)\left[\mathcal{K}_{N N}\right]-2 \xi(\phi)\left[\phi_{L}\right]\left(\phi_{N N}-\left\langle\mathcal{K}_{N N}\right\rangle \phi_{N}\right),
\end{aligned}
$$

which contains jumps and averages. However, by exploring the relation $[A]\langle B\rangle+\langle A\rangle[B]=[A B]$, the averages can be transformed away to obtain

$$
\begin{aligned}
0 & =\xi(\phi) \phi_{N}^{2} q^{A B}\left[\mathcal{K}_{A B}\right]-2 \xi(\phi) \phi_{N} \phi^{A}\left[\mathcal{K}_{N A}\right]+F^{\prime}(\phi)\left[\mathcal{K}_{N N}\right] \\
& -2 \xi(\phi)\left[\phi_{L}\right] \phi_{N N}+2 \xi(\phi) \phi_{N}\left[\phi_{L} \mathcal{K}_{N N}\right] .
\end{aligned}
$$

Equations (34)-(36) provide generalizations of the Lanczos equation, and Equation (38) a constraint on the distributional sources.

\subsection{Gauge Fixing}

At this point, it is worthwhile to remember that there is still gauge freedom in the tetrad choice. The normal vector field is autoparallel [20]

$$
N^{v} \nabla_{v} N^{\mu}=\kappa N^{\mu}
$$


with the non-affinity parameter $\kappa=\mathcal{K}_{N N}$. If the null fields are rescaled as $\bar{N}^{\mu}=e^{\alpha} N^{\mu}$ and $\bar{L}^{\mu}=e^{-\alpha} L^{\mu}$, with some function $\alpha$ defined on the hypersurface, then the non-affinity parameter changes as

$$
\bar{\kappa}=e^{\alpha}\left(N^{v} \nabla_{\nu} \alpha+\kappa\right),
$$

while

$$
\phi_{\bar{L}}=e^{-\alpha} \phi_{L}
$$

Hence,

$$
\phi_{\bar{L}} \overline{\mathcal{K}}_{\bar{N} \bar{N}}=\phi_{L}\left(N^{v} \nabla_{\nu} \alpha+\mathcal{K}_{N N}\right)
$$

It is possible to achieve

$$
\left[\phi_{\bar{L}} \overline{\mathcal{K}}_{\bar{N} \bar{N}}\right]=0
$$

through any solution of the differential equation

$$
\frac{\partial \alpha}{\partial \lambda}=-\langle\kappa\rangle-\frac{\left\langle\phi_{L}\right\rangle}{\left[\phi_{L}\right]}[\kappa]
$$

where $\lambda$ is a coordinate adapted to $N^{\mu}$, and the ratio $\left\langle\phi_{L}\right\rangle /\left[\phi_{L}\right]$ is a function on the hypersurface, being evaluated there. Hence, in this gauge, the last term of Equation (38) drops out.

\section{Discussion of the Junction Conditions}

From the $2+1$ decomposed form of the tensorial junction conditions, we may express the jumps in the components of the transverse curvature in terms of the distributional energy density, current, and isotropic pressure, as well as the jump of the transverse derivative of the scalar field and its square, as follows

$$
\begin{gathered}
{\left[\mathcal{K}_{A B}\right] q^{A B}=\frac{\rho}{F}-(\ln F)^{\prime}\left[\phi_{L}\right]+\frac{2 \xi \phi_{N}}{F}\left[\phi_{L}^{2}\right],} \\
{\left[\mathcal{K}_{N A}\right]=-\frac{j_{A}}{F}+\frac{2 \xi \phi_{N}}{F} \phi_{A}\left[\phi_{L}\right],} \\
{\left[\mathcal{K}_{N N}\right]=\frac{p}{F}+\frac{2 \xi \phi_{N}^{2}}{F}\left[\phi_{L}\right] .}
\end{gathered}
$$

Then the scalar junction equation (in the gauge where $\left[\phi_{L} \mathcal{K}_{N N}\right]=0$ ) becomes

$$
\begin{aligned}
& F^{\prime} p+\xi \phi_{N}\left(\phi_{N} \rho+2 \phi^{A} j_{A}\right) \\
= & \xi\left(2 F \phi_{N N}-F^{\prime} \phi_{N}^{2}+4 \xi \phi_{N}^{2} \phi_{A} \phi^{A}\right)\left[\phi_{L}\right]-2 \xi^{2} \phi_{N}^{3}\left[\phi_{L}^{2}\right] .
\end{aligned}
$$

There are two cases when these equations simplify considerably: (A) when there is no cubic derivative coupling $\xi=0$, and (B) when the normal derivative of the scalar field vanishes $\phi_{N}=0$. In both cases, the scalar Equation (47) shows that there is no isotropic pressure $p=0$, that the third Lanczos Equation (46) implies $\left[\mathcal{K}_{N N}\right]=0$, the second Lanczos Equation (45) gives the current as $j_{A}=-F(\phi)\left[\mathcal{K}_{N A}\right]$, and finally, the first Lanczos Equation (44) constrains the energy density as $\rho=F(\phi)\left[\mathcal{K}_{A B}\right] q^{A B}+$ $F^{\prime}(\phi)\left[\phi_{L}\right]$.

\section{Concluding Remarks}

By exploring a formalism based on a transverse null vector to the null hypersurface, we derived junction conditions across null shells in the kinetic gravity braiding theories with linear kinetic term dependence, in which the curvature and the scalar couples through a generic scalar field-dependent function. These scalar-tensor theories obey both the gravitational wave constraints and could exhibit a viable cosmological evolution through radiation-, matter-, and dark energy-dominated fixed points. 
Our formalism gives the necessary equations to discuss energetic shock waves propagating with the speed of light in these models.

The junction conditions contain the $2+1$ decomposed form of the tensorial equation, a generalization of the general relativistic Lanczos equation. This relates the jump in the transverse curvature to the distributional energy density, current, and isotropic pressure. In the relations, the jump of the transverse derivative of the scalar and its square are also involved. An additional scalar equation, without counterpart in general relativity, constrains all of these functions.

If either there is no cubic derivative coupling term $\xi=0$, or the scalar field does not change in the normal direction to the null hypersurface $\phi_{N}=0$, the junction conditions simplify considerably, leaving the possibility of a distributional source without pressure

$$
\mathscr{T}^{\mu v}=\left(F(\phi)\left[\mathcal{K}_{A B}\right] q^{A B}+F^{\prime}(\phi)\left[\phi_{L}\right]\right) N^{\mu} N^{v}-F(\phi)\left[\mathcal{K}_{N A}\right]\left(N^{\mu} e_{A}^{v}+e_{A}^{\mu} N^{v}\right),
$$

together with the geometric condition $\left[\mathcal{K}_{N N}\right]=0$. These generalize the corresponding result found for Brans-Dicke theories in the Jordan frame [26].

Author Contributions: Conceptualization, L.Á.G.; methodology, L.Á.G. and B.R.; validation, L.Á.G. and B.R.; formal analysis, B.R.; writing—original draft preparation, B.R. and L.Á.G.; writing—review and editing, B.R. and L.Á.G.; supervision, L.Á.G.; funding acquisition, L.Á.G. and B.R.

Funding: This research was funded by the Hungarian National Research Development and Innovation Office (NKFIH) in the form of grant 123996 and carried out in the framework of European Cooperation in Science and Technology (COST) actions CA15117 (CANTATA) and CA16104 (GWverse) supported by COST. During the preparation of this manuscript, B.R. was supported by the UNKP-18-3 New National Excellence Program of the Hungarian Ministry of Human Capacities.

Conflicts of Interest: The authors declare no conflict of interest.

\section{References}

1. LIGO Scientific Collaboration and Virgo Collaboration. Observation of Gravitational Waves from a Binary Black Hole Merger. Phys. Rev. Lett. 2016, 116, 061102. [CrossRef]

2. LIGO Scientific Collaboration and Virgo Collaboration. GW151226: Observation of Gravitational Waves from a 22-Solar-Mass Binary Black Hole Coalescence. Phys. Rev. Lett. 2016, 116, 241103. [CrossRef]

3. LIGO Scientific Collaboration and Virgo Collaboration. GW170104: Observation of a 50-Solar-Mass Binary Black Hole Coalescence at Redshift 0.2. Phys. Rev. Lett. 2017, 118, 221101. [CrossRef] [PubMed]

4. LIGO Scientific Collaboration and Virgo Collaboration. GW170608: Observation of a 19-Solar-Mass Binary Black Hole Coalescence. Astrophys. J. Lett. 2017, 851, L35. [CrossRef]

5. LIGO Scientific Collaboration and Virgo Collaboration. GW170814: A Three-Detector Observation of Gravitational Waves from a Binary Black Hole Coalescence. Phys. Rev. Lett. 2017, 119, 141101. [CrossRef]

6. LIGO Scientific Collaboration and Virgo Collaboration. GW170817: Observation of Gravitational Waves from a Binary Neutron Star Inspiral. Phys. Rev. Lett. 2017, 119, 161101. [CrossRef] [PubMed]

7. LIGO Scientific Collaboration and Virgo Collaboration. GWTC-1: A Gravitational-Wave Transient Catalog of Compact Binary Mergers Observed by LIGO and Virgo during the First and Second Observing Runs. arXiv 2018, arXiv:1811.12907.

8. Horndeski, G.W. Second-order scalar-tensor field equations in a four-dimensional space. Int. J. Theor. Phys. 1974, 10, 363-384. [CrossRef]

9. Deffayet, C.; Gao, X.; Steer, D.A.; Zahariade, G. From k-essence to generalized Galileons. Phys. Rev. D 2011, 84, 064039. [CrossRef]

10. Baker, T.; Bellini, E.; Ferreira, P.G.; Lagos, M.; Noller, J.; Sawicki, I. Strong constraints on cosmological gravity from GW170817 and GRB 170817A. Phys. Rev. Lett. 2017, 119, 251301. [CrossRef]

11. Ezquiaga, J.M.; Zumalacárregui, M. Dark Energy after GW170817: Dead ends and the road ahead. Phys. Rev. Lett. 2017, 119, 251304. [CrossRef] [PubMed]

12. Creminelli, P.; Vernizzi, F. Dark Energy after GW170817 and GRB170817A. Phys. Rev. Lett. 2017, 119, 251302. [CrossRef] [PubMed] 
13. Sakstein, J.; Jain, B. Implications of the Neutron Star Merger GW170817 for Cosmological Scalar-Tensor Theories. Phys. Rev. Lett. 2017, 119, 251303. [CrossRef] [PubMed]

14. Kase, R.; Tsujikawa, S. Dark energy in Horndeski theories after GW170817: A review. arXiv 2019, arXiv:1809.08735.

15. Deffayet, C.; Pujolas, O.; Sawicki, I.; Vikman, A. Imperfect Dark Energy from Kinetic Gravity Braiding. arXiv 2010, arXiv:1008.0048.

16. Kase, R.; Tsujikawa, S.; Felice, A.D. Cosmology with a successful Vainshtein screening in theories beyond Horndeski. Phys. Rev. D 2016, 93, 024007. [CrossRef]

17. Heisenberg, L.; Bartelmann, M.; Brandenberger, R.; Refregier, A. Horndeski in the Swampland. arXiv 2019, arXiv:1902.03939.

18. Israel, W. Singular hypersurfaces and thin shells in general relativity. Nouvo Cim. B 1966, 44, 1-14. [CrossRef]

19. Barrabés, C.; Israel, W. Thin shells in general relativity and cosmology: The lightlike limit. Phys. Rev. D 1991, 43, 1129-1142. [CrossRef]

20. Poisson, E. A Relativist's Toolkit: The Mathematics of Black-Hole Mechanics; Cambridge University Press: Cambridge, UK, 2004.

21. Shiromizu, T.; Maeda, K.I.; Sasaki, M. The Einstein equations on the 3-brane world. Phys. Rev. D 2000, 62, 024012. [CrossRef]

22. Gergely, L.Á. Generalized Friedmann branes. Phys. Rev. D 2003, 68, 124011. [CrossRef]

23. Gergely, L.Á. Friedmann branes with variable tension. Phys. Rev. D 2008, 78, 084006. [CrossRef]

24. Padilla, A.; Sivanesan, V. Boundary Terms and Junction Conditions for Generalized Scalar-Tensor Theories. arXiv 2012, arXiv:1206.1258.

25. Nishi, S.; Kobayashi, T.; Tanahashi, N.; Yamaguchi, M. Cosmological matching conditions and galilean genesis in Horndeski's theory. arXiv 2014, arXiv:1401.1045.

26. Racskó, B.; Gergely, L.Á. Light-Like Shockwaves in Scalar-Tensor Theories. Universe 2018, 4, 44. [CrossRef]

(C) 2019 by the authors. Licensee MDPI, Basel, Switzerland. This article is an open access article distributed under the terms and conditions of the Creative Commons Attribution (CC BY) license (http:/ / creativecommons.org/licenses/by/4.0/). 